\title{
Effects of Butyphthalide Combined with Idebenone on Inflammatory Cytokines and Vascular Endothelial Functions of Patients with Vascular Dementia
}

\author{
Fan-xing $\mathrm{Qi}^{1}$, Ying Hu2 , Li-juan Kang1, Pan Li1, Tian-chu Gao' ${ }^{1}$ and Xin Zhang \\ Department of Neurology ${ }^{1}$ / Cardiology², Baoding First Central Hospital, Baoding, China
}

\begin{abstract}
Objective: To determinate the clinical effect of butyphthalide combined with idebenone in the treatment of vascular dementia (VD) and the influence on inflammatory cytokines and vascular endothelial functions.

Study Design: Clinical comparative study.

Place and Duration of Study: Department of Neurology, Baoding First Central Hospital, from June 2017 to June 2018.

Methodology: Eighty-eight VD patients were divided into observation group (44 cases) and control group (44 cases) at random. Idebenone was given to the control group, and butyphthalide combined with idebenone was given to the observation group for 12 weeks. C-reactive protein (CRP), tumor necrosis factor $\alpha$ (TNF- $\alpha$ ), interleukin-6 (IL-6) and interleukin-1 $\beta$ (IL-1 $\beta$ ) were detected before and after the treatment to evaluate the level of serum inflammatory factors. Peripheral blood endothelial microparticles (EMPs), endothelin (ET-1), and vascular endothelial growth factor (VEGF) were detected to evaluate vascular endothelial functions. Mini-mental state examination (MMSE), clinical dementia scale $(C D R)$, and ability of daily life (ADL), were used to evaluate cognitive function, dementia degree, and self-care ability in daily life. The occurrences of adverse reactions were recorded.

Results: Before the treatment, the comparison differences in the indexes of both groups had no statistical significance $(p>0.05)$. After the treatment, the scores of CD62E+, VEGF, and MMSE of observation group rose obviously, compared with those before the treatment, and were significantly higher than those of control group $(p<0.05)$. After the treatment, the scores of IL-6, CRP, TNF- $\alpha$, IL-1 $\beta$, CD31+, CDI44+, ET-1, CDR and ADL of observation group significantly lowered, compared with those before the treatment, and were significantly lower than those of control group $(p<0.05)$. The differences in the adverse reactions of both groups had no statistical significance $(p>0.05)$.

Conclusion: Butyphthalide combined with idebenone can effectively reduce serum inflammatory factor level of VD patients, regulate vascular endothelial functions, relieve dementia degree, and improve cognitive function and daily activity ability.
\end{abstract}

Key Words: Vascular dementia, Butyphthalide, Idebenone, Serum inflammatory factor, Vascular endothelial function.

How to cite this article: Qi FX, Hu Y, Kang LJ, Li P, Gao TC, Zhang X. Effects of butyphthalide combined with idebenone on inflammatory cytokines and vascular endothelial functions of patients with vascular dementia. J Coll Physicians Surg Pak 2020; 30(1):23-27

\section{INTRODUCTION}

Vascular dementia (VD) is the second leading cause of dementia. According to etiological classification, it includes ischemia (macrovascular, small vascular, hypoperfusion), hemorrhagic, other cerebrovascular diseases (such as cerebral venous sinus thrombosis, cerebral arteriovenous malformations, etc.), and cerebrovascular diseases with Alzheimer's disease. VD has complex etiology and strong heterogeneity. 1,2 The pathophysiological mechanism of VD involves hypoperfusion, oxidative stress and inflammation, endothelial cell damage, blood-brain barrier damage and initial immune stress leading to impaired brain cell function. ${ }^{3}$ At present, there is still no specific medicine and/or uniform

Correspondence to: Ying Hu, Department of Cardiology,

Baoding First Central Hospital,Baoding 071000, China.

E-mail:jqy565@163.com

Received: May 24, 2019; Revised: October 26, 2019;

Accepted: December 19, 2019 treatment standard. Although there are cholinergic deficiencies in $A D$ patients, the therapeutic effect of anticholinergic agents on AD remains suboptimal.

Current treatments mainly focus on anti-platelet aggregation, blood pressure, cholesterol reduction and cognitive improvement. However, a single agent has no good effect in the treatment and prevention of AD.4,5 Some scholars believe the effect of the above-mentioned treatment methods to be limited, needing addition of other agents or adopting comprehensive treatment to expand the effect of comprehensive treatment. ${ }^{6}$ Butyphthalide is a new fat soluble agent, and its active ingredient is synthetic despinner DL-3-n-butylphathlide. ${ }^{7}$ Idebenone is a new brain protective agent. According to the pathophysiological mechanism of VD, it is speculated that brain protective drugs can be used in the treatment of VD.

A theoretical basis is required for clinical treatment through exploring clinical effect and safety of combined treatment of VD by butyphthalide combined with idebe-none. 
The objective of this study was to determinate the clinical effect of butyphthalide combined with idebenone in treatment of vascular dementia (VD) and the influence on inflammatory cytokines and vascular endothelial functions.

\section{METHODOLOGY}

This study was approved by the Institutional Ethics Committee of Baoding First Central Hospital, and written informed consents were obtained from all participants. VD patients treated from June 2017 to June 2018 were chosen as the subjects, and divided into observation group (basic therapy and butylphenyl peptide combined with aidibenquinone therapy, 44 cases) and control group (basic therapy and aidibenquinone therapy, 44 cases) at random. Inclusion criteria were age 45-80 years; course of disease for 1-12 months; CT/MRI verified cerebral infarction focus; conforming to diagnostic and statistical manual for mental disorders and behavioral problems, (DSM - IV), ${ }^{8}$ diagnosed by VD standards of Alzheimer's disease diagnostic and treatment centers (ADDTC); 9 who did not take any anti-dementia medicine within last one month. Exclusion criteria were hemorrhagic apoplexy; aphasia, apraxia and perceptual dysfunction; other types of dementia; severe diseases or mental disease influencing cerebral function.

Conventional therapy was performed for all patients, including blood pressure control, blood fat control, blood glucose control, and anti-platelet aggregation, with intravenous piracetam injection $(8 \mathrm{~g} / \mathrm{d})$. Based on the conventional therapy, the control group was required to take idebenone tablets orally, $30 \mathrm{mg} / \mathrm{time}, 3$ times/day. Based on this conventional therapy, butyphthalide + idebenone treatment was given to the observation group by oral administration, butyphthalide capsule $0.2 \mathrm{~g} /$ time, 3 times/day. The dose of idebenone was the same as that of control group. The treatment cycle was 12 weeks.

The basic information of patients including age, gender, course of disease, smoking and drinking history, complications (hyperlipidemia, hypertension, diabetes, peripheral vascular disease) were collected. Immunoturbidimetry was used to detect CRP level. Enzymelinked immunosorbent assay (ELISA) was applied to detect the level of IL-6, TNF- $\alpha$, and IL-1. Flow cytometry was used to detect peripheral blood EMPs and the level of specific antibodies CD62E+, CD31+ and CDI44+. ELISA was employed to detect the level of ET-1 and VEGF. MMSE was adopted to evaluate patients' dementia severity and cognitive function before and after the treatment. CDR was used to evaluate patients' dementia severity before and after the treatment. ADL was employed to evaluate patients' activity of daily life before and after the treatment.

SPSS 22.0 software was used to process the data. Enumeration data were expressed with rate (\%), and Chi-square test was used for inter-group comparison. Measurement data were expressed as mean value and standard deviation. Paired t-test was used for intragroup comparison, and independent-sample t-test was adopted for inter-group comparison. The $p<0.05$ was considered as the statistical significance level.

\section{RESULTS}

The differences of two groups in age (65.8 vs. $63.2 \mathrm{y})$, gender (male 24 vs. 21), course of disease (11.6 vs. 12.0 months), smoking (23 vs. 19), drinking history (13 vs. 17) and comorbid conditions (hyperlipidemia, hypertension, diabetes and peripheral vascular disease) had no statistical significance $(p>0.05)$. Before the treatment, the differences of various indices had no statistical significance $(p>0.05)$. After the treatment, IL-6, CRP, TNF- $\alpha$ and IL-1 levels of observation group significantly decreased, and were significantly lower than those of control group $(p<0.05)$, as shown in Table I.

Before the treatment, the comparison differences in various indexes of the patients had no statistical significance $(p>0.05)$. After the treatment, CD62E+ and

Table I: Comparison of inflammatory cytokine level $(X \pm s)(n=44 / 44)$.

\begin{tabular}{|c|c|c|c|c|c|c|c|c|}
\hline \multirow[t]{2}{*}{ Group } & \multicolumn{2}{|c|}{ IL-6 (ng/L) } & \multicolumn{2}{|c|}{$\mathrm{CRP}(\mathrm{mg} / \mathrm{L})$} & \multicolumn{2}{|c|}{ TNF- $\alpha(\mathrm{ng} / \mathrm{mL})$} & \multicolumn{2}{|c|}{ IL-1 $\beta$ (ng/L) } \\
\hline & $\begin{array}{l}\text { Before } \\
\text { treatment }\end{array}$ & $\begin{array}{c}\text { After } \\
\text { treatment }\end{array}$ & $\begin{array}{l}\text { Before } \\
\text { treatment }\end{array}$ & $\begin{array}{c}\text { After } \\
\text { treatment }\end{array}$ & $\begin{array}{l}\text { Before } \\
\text { treatment }\end{array}$ & $\begin{array}{c}\text { After } \\
\text { treatment }\end{array}$ & $\begin{array}{l}\text { Before } \\
\text { treatment }\end{array}$ & $\begin{array}{c}\text { After } \\
\text { treatment }\end{array}$ \\
\hline Experimental group & $295.35 \pm 24.51$ & $228.81 \pm 28.90^{*} \#$ & $12.19 \pm 3.18$ & $5.71 \pm 2.14^{*} \#$ & $304.41 \pm 16.80$ & $185.32 \pm 15.21^{*} \#$ & $163.72 \pm 20.51$ & $128.81 \pm 18.80^{*} \#$ \\
\hline Control group & $290.49 \pm 26.89$ & $257.88 \pm 26.69^{*}$ & $11.88 \pm 3.44$ & $7.72 \pm 2.31^{*}$ & $310.51 \pm 15.62$ & $209.84 \pm 15.42^{*}$ & $160.92 \pm 25.90$ & $145.87 \pm 19.50^{*}$ \\
\hline$t$ & 0.886 & -4.902 & 0.439 & -4.234 & -1.764 & -7.509 & 0.562 & -4.178 \\
\hline$P$ & 0.378 & $<0.001$ & 0.662 & 0.0001 & 0.081 & $<0.001$ & 0.576 & 0.0001 \\
\hline
\end{tabular}

Comparison before and after treatment: $p^{*}<0.05$; comparison with control group: $p \#<0.05$.

Table II: Comparison of MMSE, CDR and ADL scores.

\begin{tabular}{l|c|cc|cc|cc}
\hline \multirow{2}{*}{ Group } & No. & \multicolumn{2}{|c|}{ MMSE } & \multicolumn{2}{c|}{ CDR } & \multicolumn{2}{c}{ ADL } \\
\cline { 2 - 9 } & & $\begin{array}{c}\text { Before } \\
\text { treatment }\end{array}$ & $\begin{array}{c}\text { After } \\
\text { treatment }\end{array}$ & $\begin{array}{c}\text { Before } \\
\text { treatment }\end{array}$ & $\begin{array}{c}\text { After } \\
\text { treatment }\end{array}$ & $\begin{array}{c}\text { Before } \\
\text { treatment }\end{array}$ & $\begin{array}{c}\text { After } \\
\text { treatment }\end{array}$ \\
\hline Experimental group & 44 & $295.35 \pm 24.51$ & $228.81 \pm 28.90^{*} \#$ & $12.19 \pm 3.18$ & $5.71 \pm 2.14^{*} \#$ & $304.41 \pm 16.80$ & $185.32 \pm 15.21^{*} \#$ \\
\hline Control group & 44 & $290.49 \pm 26.89$ & $257.88 \pm 26.69^{*}$ & $11.88 \pm 3.44$ & $7.72 \pm 2.31^{*}$ & $310.51 \pm 15.62$ & $209.84 \pm 15.42^{*}$ \\
\hline $\mathrm{t}$ & & 0.886 & -4.902 & 0.439 & -4.234 & -1.764 & -7.509 \\
\hline $\mathrm{P}$ & & 0.378 & $<0.001$ & 0.662 & 0.0001 & 0.081 & $<0.001$ \\
\hline
\end{tabular}


VEGF of observation group rose obviously, compared with those before the treatment, and were significantly higher than those of control group $(p<0.05)$. After the treatment, the scores of CD31+, CD144+ and ET-1 observation group lowered obviously, compared with those before the treatment, and were significantly lower than those of control group $(p<0.05)$.

Before the treatment, the comparison differences in the indices of both groups had no statistical significance $(p>0.05)$. After the treatment, MMSE score of observation group rose obviously, compared with that before the treatment, and was significantly higher than that of control group $(p<0.05)$. After the treatment, CDR and ADL scores of observation group decreased obviously, compared with those before the treatment, and were significantly lower than those of control group $(p<0.05)$, as shown in Table II.

During therapy, the comparison differences in the frequency of adverse reactions such as nausea, emesis, chest distress, dizziness, liver function damage and electrocardiographic change had no statistical significance $(p>0.05)$.

\section{DISCUSSION}

In recent years, piracetam, donepezil, and nimodipine can improve VD symptoms to a certain extent, 10 and there is still no ideal specific medicine to treat VD. Many researches are confined to animal experiment stage. 11 Butylphthalide, as a neuroprotective agent for ischemic stroke, has been approved by the State Food and Drug Administration as a clinical therapeutic medicine for ischemic stroke. Butyphthalide can effectively interdict multiple pathological links of cerebral injury caused by cerebral arterial thrombosis, relieve brain cell edema, improve cerebral blood supply, promote energy metabolism and neurotransmitter transposition, with the functions of antiplatelet aggregation and anti-cerebral thrombosis formation. ${ }^{12}$ Huai et al. also showed that butylphthalide can be used to prevent and treat VD in animal experiments; and achieved good results. ${ }^{13}$ This may be related to its mechanism by upregulating the expression of phosphorylated Akt in hippocampus. Idebenone has the functions of resisting oxidation, scavenging free radical, and improving energy metabolism and cerebral blood flow. ${ }^{14}$ The curative effects of combined use of butyphthalide and idebenone in VD treatment were explored from the perspectives of serum inflammatory factors vascular endothelial functions and cognitive score.

Researches show that inflammatory cytokines participate in neuropathological damage of VD.15 In case of cerebral ischemia and reperfusion injury, endothelial cells and peripheral neurons are activated, triggering inflammatory cascade, releasing a lot of inflammatory factors, making inflammatory cytokines and metabolites gather to the damaged brain tissues, leading to cerebral vessels reocclusion in the damage zone and thus induce no-reflowing phenomenon. White blood cells can further release protease to directly damage brain tissues. ${ }^{16}$ The levels of inflammatory factors of VD patients such as IL-6, CRP, TNF- $\alpha$ and IL- $1 \beta$ were obviously higher than those of healthy persons, and anti-inflammatory therapy can effectively improve cognitive function of VD patients. 17 This study showed that the levels of IL-6, CRP, TNF- $\alpha$ and IL-1 $\beta$ of observation group declined obviously, compared with the control group, because butyphthalide can enhance energy metabolism level of brain cells, improve brain microcirculation and inhibit secondary inflammatory response and tissue damage of cerebral ischemia. ${ }^{18}$ This also indicates that there are different action mechanisms and targets between butyphthalide and idebenone, and meanwhile the two drugs have potential synergistic effect.

Hypoperfusion caused by vascular endothelial dysfunction is the core mechanism of VD attack. 19 EMPs include phenotype CDI44+ and CD31+ which lead to vascular endothelial injury and phenotype CD62E+ which promote vascular endothelial repair, which are closely related to vascular endothelial functions, 20 and participate in vascular endothelial function regulation.21-23 VEGF plays an important regulatory role for proliferation capacity of vascular endothelial cells and neovascularisation. VEGF expression increases and participates in endothelial repair and angiogenesis process. It has been found in cell and animal experiments that butylphthalide can increase the expression of growth factor, vascular endothelial growth factor, vascular endothelial growth factor receptor and vascular endothelial growth factor, which can be used to treat VD and promote angiogenesis. ET-1 is a vaso-excitor material secreted by blood vessel endothelium. Its level rises obviously in VD patients. This study showed CD31+, CDI44+ and ET-1declined obviously in the observation group, while CD62E+ and VEGF rose obviously, indicating that butyphthalide regulates vascular endothelial functions through regulating the expression of EMPs, VEGF and ET-1. The combined use of butyphthalide and idebenone can significantly improve vascular endothelial functions.

The clinical characteristics of VD are related to its mechanism. The lesions of VD are mostly found in the connective fibers from prefrontal cortex to basal ganglia and the corresponding thalamocortical connective fibers, so cognitive impairment, are characterised by attention, information processing and executive function. The damage of frontal-striatum circuit leads to Parkinson-like symptoms such as slow movement, short gait and dysarthria. Alzheimer's AD and VD overlap greatly in neuropsychiatric characteristics in having memory impairment, executive dysfunction and visualspatial dysfunction. However, many therapeutic studies 
have shown that VD is more difficult to treat than Alzheimer's disease. Cholinesterase inhibitors have little effect on VD. Butyphthalide can improve microcirculation of cerebral ischemia zone, inhibit nerve cell apoptosis, and promote the functions related to learning and memory in the hippocampus and cortical area.

Some studies have shown that SHH/PTCH1 pathway plays an important role in VD model, and butyphthalide has protective effect on cognitive impairment induced by chronic cerebral hypoperfusion. This mechanism is related to ERS and SHH/PTCH1 pathway. ${ }^{24}$ With strong anti-oxidation mechanism, idebenone can eliminate reactive oxygen and reach the purpose of delaying or stopping nerve cell degeneration. Idebenone also found that in recent years an increasing number of VD patients have been used to improve cognitive function. ${ }^{25}$ Both can improve cognitive function, and the combined use of the two has synergy or enhancement effect. After the treatment of butyphthalide and idebenone, MMSE score of observation group was significantly higher than that of control group, while CDR score and ADL score were significantly lower than those of control group. Besides, the improvement effect of dementia symptom, cognitive function, and activity of daily life was obvious. In the treatment process, adverse agent reactions of both groups were slight, indicating their safety. Future prospective studies with larger sample sizes are needed to validate this study.

\section{CONCLUSION}

The studied combination had a positive effect on VD treatment.

\section{ETHICAL APPROVAL:}

The study was approved by the Institutional Ethics Committee of Baording First Central Hospital, and ethical approvals were obtained prior to initiation of the research work.

\section{PATIENTS' CONSENT:}

Written informed consents were obtained from all participants.

\section{CONFLICT OF INTEREST:}

Authors declared no conflict of interest.

\section{AUTHORS' CONTRIBUTION:}

$F X Q, Y H$ : Designed the study and prepared the manuscript. LJK, PL: Collected and analysed clinical data.

TCG, XZ: Significantly revised the manuscript.

\section{REFERENCES}

1. Calabrese V, Giordano J, Signorile A, Laura Ontario M, Castorina S, De Pasquale $C$, et al. Major pathogenic mechanisms in vascular dementia roles of cellular stress response and hormesis in neuroprotection. J Neurosci Res 2016; 94:1588-603.

2. Doruk H, Naharci MI, Bozoglu E, Isik AT, Kilic S. The relationhip between body mass index and incidental mild cognitive impairment alzheimer's disease and vascular dementia in elderly. J Nutr Health Aging 2010; 14:834-8.

3. Iadecola $\mathrm{C}$. The pathobiology of vascular dementia. Neuron 2013; 80:844-66.

4. Auchus AP, Brashear HR, Salloway S, Korczyn AD, De Deyn PP, Gassmann-Mayer C, et al. Galantamine treatment of vascular dementia a randomized trial. Neurology 2007; 69:448-58.

5. Shepherd J, Blauw GJ, Murphy MB, Bollen EL, Buckley BM, Cobbe SM, et al. PRO spective study of pravastatin in the elderly at risk pravastatin in elderly individuals at risk of vascular disease (PROSPER) a randomised controlled. Lancet 2002; 360:1623-30.

6. Ngandu T, Lehtisalo J, Solomon A, Levälahti E, Ahtiluoto S, Antikainen $\mathrm{R}$, et al. A 2-year multidomain intervention of diet, exercise, cognitive training and vascular risk monitoring versus control to prevent cognitive decline in at-risk elderly people (FINGER): A randomised controlled trial. Lancet 2015; 385: 2255-63.

7. Chu WZ, Gong L, Xu YQ, Cai GH, Chou KC. Apolipoprotein E gene variants of Alzheimer's disease and vascular dementia patients in a community population of Nanking. Med Chem 2014; 10:783-8.

8. Cooper JE. On the publication of the diagnostic and statistical manual of mental disorders fourth edition (DSM-IV). Br J Psychiatry 1995; 166:4-8.

9. Chui HC, Victoroff JI, Margolin D, Jagust W, Shankle R, Katzman R. Criteria for the diagnosis of ischemic vascular dementia proposed by the state of California Alzheimer's disease diagnostic and treatment centers. Neurology 1992; 42:473-80.

10. Lu D, Goussev A, Chen J, Pannu P, Li Y, Mahmood A, et al. Atorvastatin reduces neurological deficit and increases synaptogenesis angiogenesis and neuronal survival in rats subjected to traumatic brain injury. $J$ Neurotrauma 2004; 21: 21-32.

11. Langdon KD, Granter-Button S, Harley CW, Moody-Corbett F, Peeling J, Corbett $\mathrm{D}$. Cognitive rehabilitation reduces cognitive impairment and normalizes hippocampal CA1 architecture in a rat model of vascular dementia. J Cereb Blood Flow Metab 2013; 33:872-79.

12. Zhang L, Lü L, Chan WM, Huang Y, Wai MS, Yew DT. Effects of DL-3-n-butylphthalide on vascular dementia and angiogenesis. Neurochem Res 2012; 37:911-9.

13. Huai $Y$, Dong $Y, X u$ J, Meng $N$, Song $C$, Li W, et al. L-3-nbutylphthalide protects against vascular dementia via activation of the Akt kinase pathway. Neural Regen Res 2013; 8: 1733-42.

14. Li XX, Zhang YY, Wang B. Research on the treatment of idebenone combined with donepezil on senile vascular dementia. Heilongjiang Med Pharm 2018; 41:49-51.

15. Economos A, Wright CB, Moon YP, Rundek T, Rabbani L, Paik MC, et al. Interleukin-6 plasma concentration associates with cognitive decline the northern Manhattan study. Neuroepidemiology 2013; 40:253-59.

16. Wan P, Wang S, Zhang Y, Lv J, Jin QH. Involvement of dopamine D1 receptors of the hippocampal dentate gyrus in spatial learning and memory deficits in a rat model of vascular dementia. Pharmazie 2014; 69:709-10.

17. Liang J, Li F, Wei C, Song $\mathrm{H}$, Wu L, Tang Y, et al. Rationale and design of a multicenter phase 2 clinical trial to investigate the 
efficacy of traditional Chinese medicine SaiLuoTong in vascular dementia. J Stroke Cerebrovasc Dis 2014; 23:2626-34.

18. Kalaria RN. Neuropathological diagnosis of vascular cognitive impairment and vascular dementia with implications for Alzheimer's disease. Acta Neuropathol 2016; 131:659-85.

19. Pantoni L, Fierini F, Poggesi A. Thrombolysis in acute stroke patients with cerebral small vessel disease. Cerebrovasc Dis 2014; 37:5-13.

20. Bruyndonckx L, Hoymans VY, Frederix G, De Guchtenaere A, Franckx $\mathrm{H}$, Vissers DK, et al. Endothelial progenitor cells and endothelial microparticles are independent predictors of endothelial function. J Pediatr 2014; 165:300-5.

21. Leslie $H$. Natalie $N$. On the origin of microparticles from plateletdust'to mediators of intercellular communication. Pulm Circ 2013; 3:329-40.
22. Van Lerssel SH, Jorens PG, Van Craenenbroeck EM, Conraads VM. The endothelium: A protagonist in the pathophysiology of critical illness focus on cellular markers. Biomed Res Int 2014; 2014:985813.

23. Markiewicz M, Richard E, Marks N, Ludwicka-Bradley A. Impact of endothelial microparticles on coagulation inflammation and angiogenesis in age-related vascular diseases. J Aging Res 2013; 2013:734509.

24. Niu XL, Jiang X, Xu GD, Zheng GM, Tang ZP, Yin N, et al. DL3-n-butylphthalide alleviates vascular cognitive impairment by regulating endoplasmic reticulum stress and the Shh/Ptch1 signaling-pathway in rats. J Cell Physiol 2019; 234:12604-14.

25. Rojas G, Demey I, Arizaga RL. Drugs used for cognitive impairment analysis of 1.5 million prescriptions in Argentina. Medicina (B Aires) 2013; 73:213-23. 